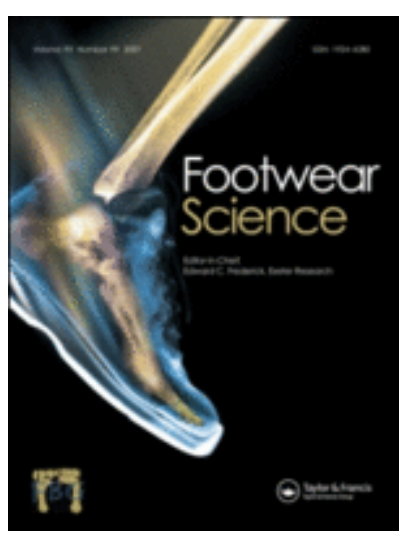

\title{
Machine learning as a tool to predict impact loading during over-ground distance running
}

\begin{tabular}{|r|l|}
\hline Journal: & Footwear Science \\
\hline Manuscript ID & TFWS-2019-0041 \\
\hline Manuscript Type: & Proceedings Abstracts FBS 2019 \\
\hline Keywords: & impact forces, wearable sensors, running, machine learning, acceleration \\
\hline
\end{tabular}

\section{SCHOLARONE Manuscripts}




\section{Introduction}

Nowadays, the use of wearable devices stimulates the prediction of running mechanics outside the lab environment (Willy, 2017). An interesting kinetic variable to predict in overground running is the repetitive impact which was identified as a possible risk factor for tibial stress fractures (Van der Worp, 2016). The proposed lab standard for impact loading is the vertical loading rate (VLR) while the use of the axial peak tibial acceleration (APTA) was verified as a moderate to good correlate for the aforementioned standard (Van den Berghe, 2019). However, we hypothesize that incorporating more advanced features of the multi-directional acceleration waveform - rather than only a 1D maximum could result in a more accurate estimation of the VLR than currently possible.

\section{Purpose of the study}

The purpose of this study was to predict the instantaneous VLR based on tri-axial shinmounted accelerometer data.

\section{Methods}

93 runners $(53 \lambda, 40$ \&) ran on a $30 \mathrm{~m}$ instrumented running track at different running speeds (from $2.55 \mathrm{~m} . \mathrm{s}^{-1}$ to $5.1 \mathrm{~m} . \mathrm{s}^{-1}$ ). They wore a backpack system connected to two tri-axial accelerometers (Sparfkun;1000 $\mathrm{Hz}$ /axis), firmly taped to each lower leg. Synched (up to 0.001s) GRFs were measured at $1000 \mathrm{~Hz}$ by two built-in force platforms $(2 \mathrm{~m}$ and $-1.2 \mathrm{~m}$, AMTI). A set of features was generated from the accelerations: (1) autogenerated statistical features: a comprehensive set of time-series features, including basic characteristics (e.g., mean, max, etc.) and complex features (e.g., CWT coefficients, ARIMA coefficients, etc.); (2)
Subject-describing features: body weight and running shoe type (neutral, stabilization, racing flats, and minimal);

(3) Application specific features: running speed and ground contact time. Using the generated features, two learning settings were trained to learn a model to estimate the instantaneous VLR:

Subject-independent: one model for all runners, trained on all runner's data. Subject-dependent: a personalized model for each runner, trained only on the data from that runner.

In both settings, Gradient Boosted Regression Trees (XGB) were used as the underlying learner. To assess the model's accuracy, a leave-one-out cross validation was used. A single-feature linear model including APTA was used to compare its predictive performance to the different learning settings. In all cases, the mean absolute error (MAE) and the coefficient of determination $\left(\mathrm{R}^{2}\right)$ were reported.

\section{Results}

The subject-dependent model learning setting outperforms (a higher $\mathrm{R}^{2}$ and a lower MAE) the predictive performance of the learner in an subject-independent model learning setting. Although, the subject-independent model setting still resulted in a better prediction compared to the single-feature model (Table 1). The mean calculation time for the latter learner was $142 \mathrm{~ms}$.

Here table 1

\section{Discussion and conclusion}

Multiple 3D acceleration features in a learning setting showed the highest accuracy in predicting the impact loading compared to 
APTA. Although resulting in the highest accuracy, a subject-dependent model is practically not applicable since it requires synched GRF and acceleration data in order to train itself. A subject-independent model, on the other hand, is more useful outside a lab setting since the model bases its predictions on data from other runners while still outperforming the single-feature model using APTA.

This machine learning approach, based on bilateral lower leg accelerations, provides a useful way to predict the instantaneous VLR during over-ground distance running. Furthermore, its short calculation time offers possibilities towards design of wearable realtime feedback applications on impact loading during distance running outside the lab.

\section{References}

Willy, R. (2018). Innovations and pitfalls in the use of wearable devices in the prevention and rehabilitation of running related injuries. Physical Therapy in Sport, 29, 26 - 33. Van den Berghe, P., Six, J., Gerlo, J., Leman, M., De Clercq, D. (2019). Validity and reliability of peak tibial accelerations as realtime measure of impact loading during overground rearfoot running at different speeds. Journal of Biomechanics. Advance online publication. doi.org/10.1016/j.jbiomech.2019.01.039. van der Worp, H., Vrielink J.W., \& Bredeweg, S.W. (2016). Do runners who suffer injuries have higher vertical ground reaction forces than those who remain injury-free? A systematic review and meta-analysis. British Journal of Sports Medicine, 50(8), $450-457$. 


\begin{tabular}{lccc}
\hline & SIM & SDM & \multirow{2}{*}{ APTA } \\
& XGB & XGB & \\
\hline $\mathrm{MAE}$ & 12.47 & 5.39 & \multirow{2}{*}{19.85} \\
$\left(\mathrm{BW}^{*} \mathrm{~s}^{-1}\right)$ & \pm 7.90 & \pm 2.04 & \\
$\mathrm{R}^{2}$ & 0.79 & 0.95 & 0.58 \\
\hline
\end{tabular}

